\title{
Assessing the Correlation Between Autoimmune Bullous Disease Quality of Life Questionnaires (ABQOL, TABQOL, DLQI) and Disease Severity Indices in Pemphigus and Bullous Pemphigoid
}

\section{Shan Cao}

Shandong Provincial Institute of Dermatology and Venereology

Lulu Sun

Shandong Provincial Institute of Dermatology and Venereology

Zhongxiang Shi

Shandong Provincial Institute of Dermatology and Venereology

Baoqi Yang ( $\sim$ baoqiyang@126.com )

Shandong Provincial Institute of Dermatology and Venereology https://orcid.org/0000-0003-35217130

\section{Furen Zhang}

Shandong Provincial Institute of Dermatology and Venereology

\section{Research}

Keywords: pemphigus, bullous pemphigoid, disease severity, quality of life, measurement tools, association

Posted Date: December 15th, 2020

DOI: https://doi.org/10.21203/rs.3.rs-127372/v1

License: (c) (1) This work is licensed under a Creative Commons Attribution 4.0 International License. Read Full License 


\section{Abstract}

Background: Pemphigus and bullous pemphigoid (BP) are autoimmune blistering diseases (AIBDs) that affect the skin and mucous membranes, and adversely impact quality of life (QOL). Few studies have assessed the correlation between the severity of disease and QOL in patients with pemphigus and BP.

Objectives: To identify the correlation between the severity of AIBDs and QOL using the Autoimmune Bullous Disease Quality of Life (ABQOL), Treatment Autoimmune Bullous Disease Quality of Life (TABQOL), and Dermatology Life Quality Index (DLQI) questionnaires in Chinese patients with pemphigus and $\mathrm{BP}$ at baseline, and after 1,3 and 6 months of treatment.

Methods: Pemphigus and BP patients were invited to complete the ABQOL, TABQOL, and DLQI questionnaires. We measured the pemphigus disease area index (PDAl), autoimmune bullous skin disorder intensity score (ABSIS), and antibodies of desmoglein1,desmoglein3 (DSG1/DSG3) for pemphigus; and the bullous pemphigoid disease area index (BPDAI), ABSIS, and antibodies of BP180/ $\mathrm{BP} 230$ for BP as disease severity indices. The correlations between the severity of disease and QOL were analyzed by Spearman's correlation coefficient (r).

Results: Eighty-five patients were included: 55 with pemphigus and 30 with BP. The pemphigus subtypes included pemphigus vulgaris ( $\mathrm{PV}, n=32$ ), pemphigus foliaceus ( $\mathrm{PF}, n=22)$, and paraneoplastic pemphigus (PNP, $n=1$ ). We found significantly strong correlations between QOL (ABQOL, TABQOL, DLQI) and severity of disease (PDAl/BPDAl, ABSIS) with $(r=0.87,0.77,0.83 ; r=0.86,0.73,0.80)$ for pemphigus and $\mathrm{BP}$, respectively. Mild or strong correlations were also observed between $\mathrm{QOL}$ and antibody titers in pemphigus with DSG1/DSG3( $r=0.32 / 0.36)$ and BP with BP180/BP230( $r=0.73 / 0.17)$ respectively.

Conclusion: The QOL of patients with pemphigus and BP decreased with increased severity of the AIBDs. As the disease severity descended, so the QOL improved. The QOL indices should be used in clinical trials and to manage patients' treatment, especially during the active disease stage, despite the mild correlation observed after 1 month of treatment.The PDAl to be better at assessing disease severity than the ABSIS in patients with pemphigus, and ABSIS are better than BPDAI in BP patients for correlation with the QOL indices.

\section{Introduction}

Pemphigus and bullous pemphigoid (BP) are the most common, life-threatening chronic autoimmune blistering diseases (AIBDs) that affect the skin and mucous membranes[1, 2]. Pemphigus includes two main pemphigus variants of pemphigus vulgaris(PV) and pemphigus foliaceus(PF), $P V$ is characterised by painful oral erosions and presented as enanthema and erosions, while PF is originated with exclusive skin lesions.Pemphigus erythematosus(PE) is a clinical subtype of PF,which presentes as erythema,erosions and so-called puff pastry-like scaling on the face,back,and trunk. Paraneoplastic pemphigus (PNP) as rare pemphigus variants accounts for about 5\%of pemphigus[1]. Pneumonia and septicaemia were the most frequent causes of death,followed by cardiovascular diseases and peptic 
ulcer disease.Skin barrier, breakdown and infection of the oral cavity thereby affecting nutrition, and the need for steroids and immunosuppressants to treat the disease can present financial difficulties, all resulting in significant physical and psychological burdens for the patients $[3,4]$. Studies have shown that pemphigus and BP adversely affect a patient's quality of life (QOL), by impacting their emotions, physical health, and social functioning $[4,5]$. With different clinical manifestations, the pemphigus subtypespemphigus vulgaris (PV), pemphigus foliaceus (PF), and paraneoplastic pemphigus (PNP) $[1,6]$-have varying effects on patients' QOL.

The impact of the disease on QOL is now receiving more attention from doctors, and studies have been conducted to develop new QOL tools, such as the Autoimmune Bullous Disease Quality of Life (ABQOL), Treatment Autoimmune Bullous Disease Quality of Life (TABQOL), and Dermatology Life Quality Index (DLQI) questionnaires to evaluate patients and their QOL[3-5, 7, 8]. Some studies have also investigated the correlations between disease severity and QOL in different disease profiles[9]. A recent study by Krain et al.[10]showed that the autoimmune bullous skin disorder intensity score (ABSIS) was weaker than the pemphigus disease area index (PDAl) in 50 patients with mild pemphigus, and did not incorporate data on comorbid conditions. However, this study did not include all data-points, and did not investigate patients with various pemphigus subtypes, including PV,PF and PNP.

The aim of this prospective study was to investigate the correlation between disease severity and QOL in Chinese patients with pemphigus (including PV, PF, and PNP) and BP at baseline, and after 1, 3 and 6 months of treatment, with the aim to reduce administration of glucocorticoids and immunosuppressants. A better understanding of this relationship could help in managing the treatment of patients with pemphigus and BP.

\section{Methods}

At the Shandong Provincial Hospital for Skin Disease, over the period January 2019 to July 2020, we obtained written consent from patients with typical clinical presentation and histological testing consistent with pemphigus, and diagnosis of BP by direct immunofluorescence (DIF), indirect immunofluorescence (IIF) assay (using monkey esophagus as substrate), and enzyme-linked immunosorbent assay (ELISA). Pemphigus was verified by ELISA for DSG1 and DSG3; and manifestations; PV was detected by DSG1 and DSG3,which preferentially generate anti-DSG3 autoantibodies, while PF is only associated DSG1.BP was verified by BP180-ELISA and BP230-ELISA.A history of tumor and polymorphous mucocutaneous eruption, besides IIF assay, verified PNP patients[1].

PDAl scores range from 0 to 263 points, with disease classified as mild $(0 \sim 8)$, moderate $(9 \sim 24)$, or severe $(>25)[11-14]$. The total BPDAl activity score ranges from 0 to 360 including the main subcomponents: blisters/erosions, cutaneous urticarial /erythema, and mucosal blisters/erosions, with higher scores indicating greater disease activity or damage $[15,16]$. The ABSIS score, obtained by scoring the quality of the skin lesion, indicates both disease activity and damage, with scores ranging from 0 to 206[12-18]. AlBDs were assessed by QOL measurement tools including the ABQOL, TABQOL, and DLQI. 
The higher the scores, the worse the QOL indices. The ABQOL and TABQOL questionnaires each consist of 17 questions for patients with AIBDs, covering topics such as pain, itch, and work discrimination[1921]. The DLQI is a 10-question survey, with possible scores ranging from 0 to 30 points[4]. All patients diagnosed with pemphigus or $\mathrm{BP}$, or relapsed for the first time (baseline), were invited to participate in follow-up visits. Patients completed the ABQOL, TABQOL, and DLQI of QOL forms and were assessed by a doctor using the PDAI/BPDAI and ABSIS scoring systems at baseline, and after 1, 3 and 6 months of treatment.

Pemphigus and BP patients accompanying comorbid conditions are also need to assess whether there is a significant difference in scores between the 'complication' group and the 'non-complication' group.

\section{Statistical Analysis}

The correlations between the severity of the disease(PDAI/BPDAl,ABSIS,antibody titers) and QOL(ABQOL,TABQOL,DLQI) were analyzed using Spearman's correlation coefficient $(r)$ for all components and a mixed linear model for changes of the average trend. Spearman correlation coefficient cut-offs for the absolute value of $0-0.19,0.2-0.39,0.4-0.59,0.6-0.79$, and $0.8-1.0$ corresponded to weak, mild, moderate, strong, and very strong, respectively[22]. Statistical significance was defined by a $P$-value < 0.05 . SPSS version 24 was used to conduct the analyses.

\section{Results}

A total of 85 patients, including $55(65 \%)$ with pemphigus and $30(35 \%)$ with BP were recruited to the study. The pemphigus group included $32(58 \%)$ with PV, 22(40\%) with PF, and one (2\%) with PNP after 3 months of treatment, and $65(76 \%)$ of the patients(35 pemphigus and $30 \mathrm{BP}$ ) completed the 6-month follow-up. The mean age was 56.2 (range $28-82$ ) years and the male-to-female ratio was 5:6. Table 1 illustrates the total demographic and disease characteristics of the patients with pemphigus and BP.

Figure 1 show the mixed linear model for the changes in median QOL (ABQOL,TABQOL,DLQI) indices and disease severity indices (PDAI/BPDAl, ABSIS) in 85 Chinese patients with pemphigus and BP at baseline, and after 1 and 3 months of treatment. Figure 2 show the changes in 65 patients with pemphigus and BP at baseline, and after 1, 3 and 6 months of treatment.

Thirty (55\%) patients with pemphigus and 19 (63\%) with BP had accompanying comorbid conditions, including hypertension, diabetes, osteoporosis, onychomycosis, syphilis, hyperlipidemia, porokeratosis, hypertension, cervical spondylosis, tuberculosis, epilepsy, and nephrotic syndrome. We did not find a significant difference in scores between the 'complication' group (disease severity score $118.30 \pm 51.88$, QOL score $62.82 \pm 19.72$ ) and the 'non-complication group (disease severity score $116.92 \pm 49.28$, QOL score $61.56 \pm 21.95)$, but the correlations between QOL and disease severity were strong in both groups ( $r$ $=1.00$ ). 
The median disease severity (median PDAI/BPDAl, ABSIS) scores are shown in Table 2. The median scores of PDAI/BPDAl, ABSIS are shown for the pemphigus and BP patients at baseline all were $\geq 45$.

We found a strong correlation between disease severity and QOL in all $(n=85)$ patients $(r=0.87,0.77$, $0.83)$, the pemphigus group $(r=0.84,0.81,0.81)$ and the BP group $(r=0.94,0.77,0.89)$, using PDAI/BPDAI, ABSIS with ABQOL, TABQOL, and DLQI, respectively (Table 3). The only exception was the correlation between disease severity and $\mathrm{QOL}$ in the pemphigus group after 1 month of treatment: PDAI with ABQOL $(r=0.28)$, TABQOL $(r=0.33)$, DLQI $(r=0.35)$; ABSIS with ABQOL $(r=0.25)$, TABQOL $(r=0.24)$, and DLQI $(r=0.36)$. There was a weak correlation between ABSIS and TABQOL $(r=0.36)$ in the BP group after 1 month of treatment. The correlations for the pemphigus subgroups, after 1 month of treatment, between PDAl and ABQOL $(r=0.19)$, TABQOL $(r=0.15)$, DLQI $(r=0.23)$ for PV, and PDAl and ABQOL $(r=$ $0.38)$ for PF at Month 1. However, the ABSIS and ABQOL ( $r=0.06)$, TABQOL $(r=-0.015)$, DLQI $(r=0.13)$ only showed weak correlations for the patients with PV after 1 month. There were no correlations $(r=0)$ in the pemphigus group after 6 months of treatment, but mild to strong correlations between BPDAI and ABQOL,TABQOL,DLQI $(r=0.31,0.65,0.50)$, and ABSIS and ABQOL,TABQOL,DLQI $(r=$ $0.48,0.80,0.45$,respectively) in BP group after 6 months of treatment.

The correlations between BPDAI score (erosion/blisterlerythema/urticaria) with QOL indices(ABQOL,TABQOL,DLQI) are shown in Table 4. Moderate and strong correlations $(r=0.58,0.56$, and 0.67) between BPDAI erosion/blister-score and ABQOL,TABQOL,DLQI were seen at baseline.There are moderate and strong correlations $(r=0.73,0.56,0.53)$ between the BPDAl erythema/urticaria-score and ABQOL,TABQOL,DLQI at Month1. There were weak correlations between BPDAI mucosal blister/erosionsscore and $A B Q O L, T A B Q O L$,and DLQI at baseline,month1,month3 and month6. For patients with PV and $\mathrm{PF}$, correlations between the QOL score and the changes in disease severity from baseline and 1 and 3 months after treatment are shown in Table 5. All PDAI/ABSIS scores in PV and PF show mild and strong correlations with $A B Q O L, T A B Q O L$, and DLQI except ABSIS with TABQOL at month 1 in PV patients.

The changes in antibody (DSG1/DSG3, BP180/BP230) levels showed mild or strong correlations ( $r=$ $0.32 / 0.36$ and $r=0.73 / 0.17$, respectively) with the QOL indices in the pemphigus and BP groups, respectively. Mild or strong correlations were seen with antibody levels DSG1 and ABQOL, TABQOL, DLQI in pemphigus $(r=0.33,0.30,0.32)$, and DSG3 with ABQOL, TABQOL, DLQI $(r=0.37,0.34,0.32$, respectively). Additionally, mild or strong correlations were seen in BP between antibody levels BP180 and ABQOL, TABQOL, DLQI $(r=0.73,0.61,0.73)$, and BP230 with ABQOL, TABQOL, DLQI $(r=0.11,0.21,0.19$, respectively).

\section{Discussion}

AIBDs are severe, chronic diseases with an enduring course that requires long-term treatment, resulting in decreased QOL[1, 2]. Previous studies recruited 70 patients with AIBDs to develop a 45-item pilot ABQOL and TABQOL questionnaire to assess QOL, and concluded that the ABQOL may serve as an end-point in clinical trials with adequate validity and reliability $[8,19]$. Previous studies have examined QOL in patients 
with AIBDs using various measurement tools, such as the ABQOL in USA[3], the Chinese version of $A B Q O L$ and TABQOL[7, 8] and the Polish versions of the ABQOL and TABQOL[20], thus verifying the efficacy of the Chinese version of the QOL questionnaire for this study.

Hébert et al.[11] concluded-after studying 50 patients-that the PDAI and ABSIS are effective tools to assess pemphigus activity. Ferries et al.[21] assessed the correlation between the severity of disease (PDAI/BPDAI) and QOL (ABQOL, TABQOL, DLQI, Skindes-29) in pemphigus and BP patients, with different assessment tools compared with our study. The previous studies had some limitations, such as the effect of comorbid conditions, follow-up visits, and the addition of more disease severity tools, such as antibody titers[23]. In this study, we described and evaluated the correlation between disease severity (PDAI/BPDAl, ABSIS) and QOL (ABQOL, TABQOL, DLQI) at baseline, and after 1, 3, and 6 months of treatment in Chinese patients with pemphigus and BP to exclude these limitations.

Our median PDAI/BPDAIIABSIS score and IQR in table 2 illustrated the pemphigus and BP patients can be applicable to patients with moderate to severe disease.Strong correlations were found between the QOL indices (ABQOL,TABQOL,DLQI) and the disease severity indices (PDAI/BPDAI,ABSIS), showing a decrease in QOL with increased pemphigus and BP disease severity. A notable exception was the mild correlation between disease severity indices and QOL after 1 month of treatment. The mild and moderate correlations were observed between the PDAl and ABQOL, TABQOL in the pemphigus (PV, PF) group, and between the BPDAl and TABQOL in the BP group. The patients with pemphigus (including PV and PF) tended to recover after 1 month of treatment, but the administration of steroids and immunosuppressants resulted in diet and health recovering more slowly, leading to adverse emotions and financial worries, resulting in high QOL scores, even though the disease severity had decreased. All of the indices showed a mild or strong correlation with each other and the PDAI was greater than the ABSIS in patients with pemphigus, which is consistent with previous studies[21]; The ABSIS was greater than the BPDAl in patients with BP. The correlation between QOL and the severity of pemphigus after 6 months of treatment was 0 in the pemphigus group, which could be due to pemphigus being more severe than $\mathrm{BP}$, and requiring longer treatment, with greater economic pressure. For most pemphigus patients, after 6 months of treatment the condition is under control and at the stage of drug reduction, which is why there was no obvious correlation between QOL and disease severity in pemphigus patients at Month 6 .

The correlations of the subcomponents of BPDAI with ABQOL, TABQOL, and DLQI are varied. The correlations with the BPDAI erosion/blister score at baseline are the "strongest" and the correlations with BPDAl erythema/urticaria at month 1 are the "strongest". This is considered to be the result of treatment between baseline and month 1. The correlations with BPDAI mucosal blister/erosions score are all weak, due to the BP involved mucosal are few.

Dsg1 and Dsg3 are the major target antigens in pemphigus, and BP180 and BP230 are also target antigens in BP; Both autoantibodies are related to disease severity $[1,2,24]$.As predicted, we found that the antibody titers had both mild and strong correlations with QOL. In our study,we found that mild and strong correlations were also observed between QOL and antibody titers in pemphigus with DSG1 $(r=$ 
0.32),DSG3( $r=0.36)$ and BP with BP180( $r=0.73), B P 230(r=0.17)$ respectively, In particular DSG3 (for pemphigus) and BP180 (for BP) both showed "strong" correlations, which was also explained from the other side the correlation between disease severity with QOL and this phenomenon is consistent with the main pathogenic antibodies of clinical diseases. When autoantibodies decreased in the pemphigus and BP patients, the QOL scores also decreased, which shows that the improvement in the disease severity is particularly important for the improvement of the QOL. Our study illustrates that it is important for patients with pemphigus and BP to control any complications during treatment, although we found no difference between the complication and non-complication groups.

Our study also compared the PDAI/BPDAI and ABSIS using the Spearman's correlation coefficient ( $r$ ). We found the PDAI was better at assessing disease severity than the ABSIS in patients with pemphigus, based on the correlation with the QOL indices. However, the ABSIS are prior to BPDAI in terms of correlation with the QOL indices. We believe the reason for this is that the BPDAl includes the main subcomponents: blisters/erosions, cutaneous urticaria/erythema, and mucosal blisters/erosions, and are also include objective measurement of pruritus with 30 scores, which may mean that the BPDAl scores are less sensitive than ABSIS.

In the future, our study needs to be repeated at a multicenter institution where more pemphigus and BP patients can be recruited, including other rare types of AIBDs patients. Our study would benefit from the addition of Skindex-S and Skindex-29 to the QOL tools to evaluate the correlations between disease severity and QOL. Despite its limitations, we have described the correlation between QOL (ABQOL, TABQOL, DLQI) and disease severity (PDAI/BPDAI, ABSIS) in Chinese patients with pemphigus (PV, PF) and $\mathrm{BP}$ at baseline, and after 1, 3 and 6 months of treatment.

\section{Conclusion}

We identified the correlation between the disease severity index (PDAl/BPDAl, ABSIS) and QOL (ABQOL, TABQOL, DLQI). QOL should be included in clinical trials and in management of the treatment of Chinese patients, especially when the disease is active, despite the mild correlation observed at 1 month. Patients families and clinical staff should pay more attention to QOL assessments. Future studies should include the Skindex-S subscale of the Chinese version of the Skindex-29 as a QOL tool to assess disease severity indices, and the sample size should be expanded to elucidate the utility of the various disease severity tools with QOL.

\section{Abbreviations}

BP:Bullous pemphigoid; AIBDs:Autoimmune blistering diseases; QOL:Quality of life; ABQOL:Autoimmune Bullous Disease Quality of Life; TABQOL:Treatment Autoimmune Bullous Disease Quality of Life; DLQI:Dermatology Life Quality Index; PDAl:Pemphigus disease area index; ABSIS:Autoimmune bullous skin disorder intensity score; DSG1:Desmoglein1; DSG3:desmoglein3; BPDAl:Bullous pemphigoid disease area index; PV:Pemphigus vulgaris; PF:Pemphigus foliaceus; PNP:Paraneoplastic pemphigus; DIF:Direct 
immunofluorescence; IIF:Indirect immunofluorescence assay; ELISA:Enzyme-linked immunosorbent assay.

\section{Declarations}

\section{ACKNOWLEDGMENTS}

The authors are grateful to all participants and assessors for their contribution.

\section{Funding}

The study did not receive funding.

\section{Availability of data and materials}

The dataset supporting the conclusions of this article may be available upon request from the lead author to ensure any changes to the project do not invalidate the project's ethical approval in accordance with the Ethics Committee of Shandong Provincial Hospital for Skin Diseases approval letter.

\section{Authors' contributions}

BY designed the study; SC developed the idea in consultation with BY and was responsible for statistical analyses ; LS, ZS and FZ were responsible for data collection.

All authors read and approved the final manuscript.

\section{Competing interests}

The authors declare that they have no competing interests.

\section{Consent for publication}

Not applicable.

\section{Ethics approval and consent to participate}

The study was conducted in accordance with the Declaration of Helsinki. The protocol was approved by the Ethics Committee of Shandong Provincial Hospital for Skin Diseases. All participants signed the written consent form.

\section{References}

1. Schmidt E, Kasperkiewicz M, Joly P. Pemphigus. Lancet 2019;394(10201):882-94.

2. Kayani M, Aslam AM. Bullous pemphigoid and pemphigus vulgaris. BMJ 2017;8(357):j2169. 
3. Sebaratnam DF, Okawa J, Payne A, Murrell DF, Werth VP. Reliability of the autoimmune bullous disease quality of life (ABQOL) questionnaire in the USA. Qual Life Res 2015;24(9):2257-60.

4. Sebaratnam DF, McMillan JR, Werth VP, Murrell DF. Quality of life in patients with bullous dermatoses. Clin Dermatol 2012;30(1):103-7.

5. Ghodsi SZ, Chams-Davatchi C, Daneshpazhooh M, Valikhani M, Esmaili N. Quality of life and psychological status of patients with pemphigus vulgaris using Dermatology Life Quality Index and General Health Questionnaires. J Dermatol 2012;39(2):141-4.

6. Hammers CM, Stanley JR, Schmidt E. Mechanisms of disease: pemphigus and bullous pemphigoid. Annu Rev Pathol 2016;11:175- 97.

7. Yang B, Chen G, Yang Q, Yan X, Zhang Z, Murrell DF, et al. Reliability and validity of the Chinese version of the autoimmune bullous disease quality of life(ABQOL) questionnaire. Health Qual Life Outcomes2017;15(1):31.

8. Chen G, Yang B, Zhang Z, Yang Q, Yan X, Murrell DF,et al. Chinese version of the treatment of autoimmune bullous disease quality of life questionnaire: Reliability and validity. Indian J Dermatol Venereol Leprol2018;84(4):431-6.

9. Putterman E, Patel DP, Andrade G, Harfmann KL, Hogeling M, Cheng CE,et al. Severity of disease and quality of life in parents of children with alopecia areata, totalis, and universalis: A prospective, crosssectional study. J Am Acad Dermatol 2019;80(5):1389-94.

10. Krain RL, Kushner CJ, Tarazi M, Gaffney RG, Yeguez AC, Zamalin DE,et al. Assessing the Correlation Between Disease Severity Indices and Quality of Life Measurement Tools in Pemphigus. Front Immunol 2019;10:2571.

11. Hébert V, Boulard C, Houivet E, Duvert Lehembre S, Borradori L,et al. Large International Validation of ABSIS and PDAI Pemphigus Severity Scores. J Invest Dermatol 2019;139(1):31-7.

12. Boulard C, Duvert Lehembre S, Picard-Dahan C,Kern JS, Zambruno G, Feliciani C, et al. Calculation of cut-off values based on the Autoimmune Bullous Skin Disorder Intensity Score (ABSIS) and Pemphigus Disease Area Index (PDAl) pemphigus scoring systems for defining moderate, significant and extensive types of pemphigus. Br J Dermatol 2016;175(1):142-9.

13. Murrell DF, Dick S, Ahmed AR, Amagai M, Barnadas MA, Borradori L,et al. Consensus statement on definitions of disease, end points, and therapeutic response for pemphigus. J Am Acad Dermatol 2008;58(6):1043-6.

14. Rahbar Z, Daneshpazhooh M, Mirshams-Shahshahani M, Esmaili N, Heidari K, Aghazadeh N,et al. Pemphigus disease activity measurements: pemphigus disease area index, autoimmune bullous skin disorder intensity score, and pemphigus vulgaris activity score. JAMA Dermatol 2014;150(3):266-72.

15. Murrell DF, Daniel BS, Joly P, Borradori L, Amagai M, Hashimoto T,et al. Definitions and outcome measures for bullous pemphigoid: recommendations by an international panel of experts. J Am Acad Dermatol 2012;66(3):479-85.

16. Wijayanti A, Zhao CY, Boettiger D, Chiang YZ, Ishii N, Hashimoto T,et al. The Reliability, Validity and Responsiveness of Two Disease Scores (BPDAI and ABSIS) for Bullous Pemphigoid: Which One to 
Use? Acta Derm Venereol 2017;97(1):24-31.

17. Pfütze $M$, Niedermeier A, Hertl M, Eming R. Introducing a novel Autoimmune Bullous Skin Disorder Intensity Score (ABSIS) in pemphigus. Eur J Dermatol. 2007;17(1):4-11.

18. Daniel BS, Hertl M, Werth VP, Eming R, Murrell DF. Severity score indexes for blistering diseases. Clin Dermatol 2012;30(1):108-13.

19. Sebaratnam DF, Hanna AM, Chee SN, Frew JW, Venugopal SS, Daniel BS,et al. Development of a quality-of-life instrument for autoimmune bullous disease: the Autoimmune Bullous Disease Quality of Life questionnaire. JAMA Dermatol 2013;149(10):1186-91.

20. Kalinska-Bienias A, Jakubowska B, Kowalewski C, Murrell DF, Wozniak K. Measuring of quality of life in autoimmune blistering disorders in Poland. Validation of disease-specific Autoimmune Bullous Disease Quality of Life (ABQOL) and the Treatment Autoimmune Bullous Disease Quality of Life (TABQOL) questionnaires. Adv Med Sci2017;62(1):92-6.

21. Ferries L, Gillibert A, Duvert-Lehembre S, Corbaux C, Alexandre M, Prost-Squarcioni C,et al.Sensitivity to change and correlation between the autoimmune bullous disease quality-of-life questionnaires ABQOL and TABQOL, and objective severity scores.Br. J. Dermatol 2020;183(5):944-5.

22. Punekar YS, Riley JH, Lloyd E, Driessen M, Singh SJ. Systematic review of the association between exercise tests and patient-reported outcomes in patients with chronic obstructive pulmonary disease. Int J Chron Obstruct Pulmon Dis 2017; 12(2):2487-506.

23. Tjokrowidjaja A, Daniel BS, Frew JW, Sebaratnam DF, Hanna AM, Chee S, et al. The development and validation of the treatment of autoimmune bullous disease quality of life questionnaire区a tool to measure the quality of life impacts of treatments used in patients with the autoimmune blistering disease.Br J Dermatol 2013;169(5):1000-6.

24. Schmidt E, Dähnrich C, Rosemann A, Probst C, Komorowski L, Saschenbrecker S, et al. Novel ELISA systems for antibodies to desmoglein 1 and 3: correlation of disease activity with serum autoantibody levels in individual pemphigus patients. Exp Dermatol 2010;19(5):458-63.

\section{Tables}

Table 1 Characteristics of patients with pemphigus and BP at follow-up after 3 and 6 months of treatment. 


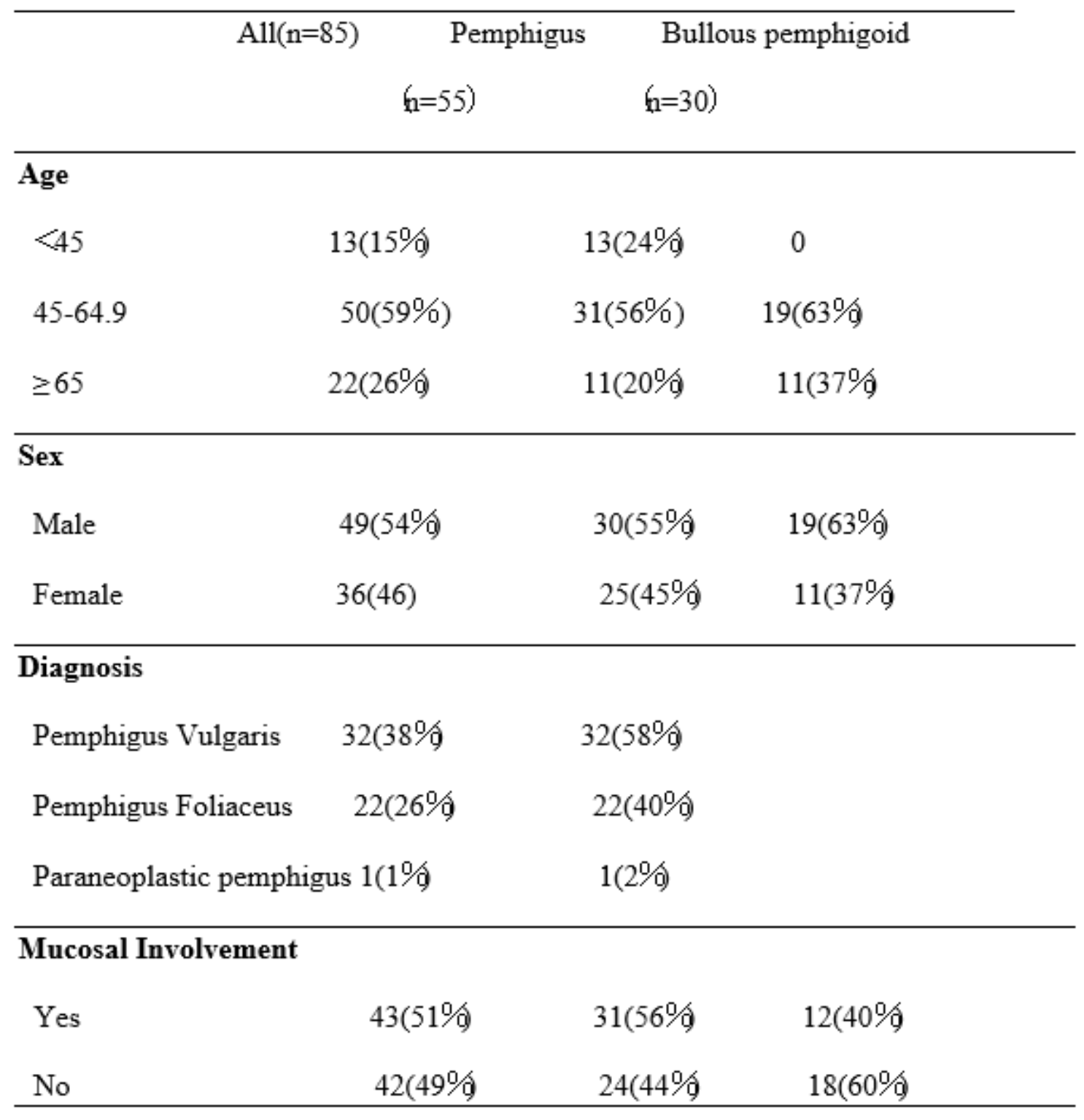

Table 2 Median PDAI/BPDAIIABSIS score and IQR in the pemphigus and BP groups. 


\begin{tabular}{|c|c|c|c|c|}
\hline & \multicolumn{2}{|c|}{ pemphigus Group $(n=55)$} & \multicolumn{2}{|c|}{ BP Group $(n=30)$} \\
\hline & Median & score & Median score & IQR \\
\hline PDAI/BPDAI & 21.00 & $8.5-45.5$ & 11.00 & $1.25-36.50$ \\
\hline Baseline & 58.00 & $35.0-73.0$ & 71.25 & $41.50-83.00$ \\
\hline Month1 & 22.00 & $14.00-32.00$ & 23.00 & $16.50-32.63$ \\
\hline Month3 & 6.00 & $2.00-11.00$ & 9.00 & $4.50-11.00$ \\
\hline Month6(n=35) & 0.00 & 0 & 0 & $0-0.63$ \\
\hline ABSIS & 15.50 & $6.00-35.00$ & 10.75 & $1.50-35.38$ \\
\hline Baseline & 49.00 & $28.00-75.00$ & 69.50 & $45.13-80.88$ \\
\hline Month1 & 20.50 & $10.50-29.00$ & 20.25 & $14.75-29.00$ \\
\hline Month3 & 4.50 & $1.00-8.00$ & 8.25 & $3.88-10.50$ \\
\hline Month6 & 0.00 & 0 & 0.00 & $0.00-0.63$ \\
\hline
\end{tabular}

PDAI, Pemphigus Disease Area Index; ABSIS, Autoimmune Bullous Skin Disorder Intensity Score.

Table 3 Correlation using Spearman's correlation coefficient ( $r$ ) of the change in QOL score with the change in disease severity in the pemphigus and BP groups. 


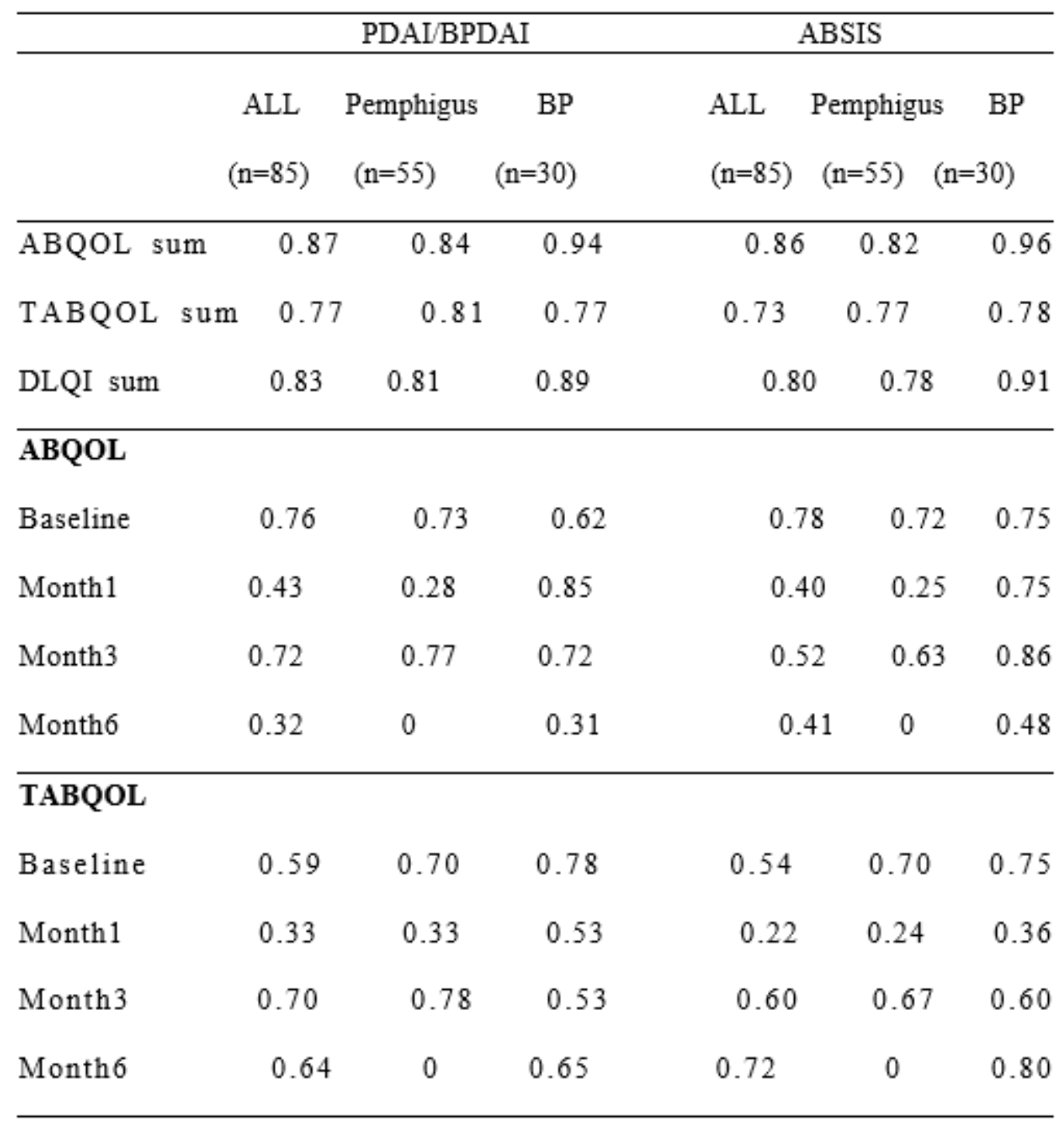

\section{DLQI}

\begin{tabular}{lcccccc} 
Baseline & 0.69 & 0.67 & 0.78 & 0.67 & 0.62 & 0.78 \\
Month1 & 0.36 & 0.35 & 0.55 & 0.34 & 0.36 & 0.54 \\
Month3 & 0.72 & 0.80 & 0.49 & 0.63 & 0.70 & 0.66 \\
Month6 & 0.54 & 0 & 0.50 & 0.50 & 0 & 0.45 \\
\hline
\end{tabular}

ABQOL, Autoimmune Bullous Disease Quality of Life; TABQOL, Treatment Autoimmune Bullous Disease Quality of Life; DLQI, Dermatology Life Quality Index.

Table 4 The correlations between BPDAI scores (erosion/blister $\square$ erythema/urticaria $\square$ mucosal blisters/erosions) with QOL indices(ABQOL/TABQOL/DLQI). 

ABQOL
TABQOL
DLQI

\section{BPDAI erosion/blister score}

Baseline

0.58

0.56

0.67

Month1

0.28

0.03

0.37

Month3

0

0

0

Month6

0

0

0

\section{BPDAI erythema/urticaria score}

Baseline

0.28

0.28

0.18

Month1

0.73

0.56

0.53

Month3

0.50

0.21

0.32

Month6

0

0

0

\section{BPDAI mucosal blisters/erosions score}

\begin{tabular}{lccc} 
Baseline & 0.06 & 0.12 & 0.08 \\
Month1 & 0.16 & -0.11 & -0.08 \\
Month3 & 0.18 & 0.24 & 0.19 \\
Month6 & 0 & 0 & 0 \\
\hline
\end{tabular}

Table 5 Correlations between Spearman's correlation coefficient ( $r$ ) of patients with PV, PF in QOL score and the change in disease severity from baseline, and 1 and 3 months after treatment. 


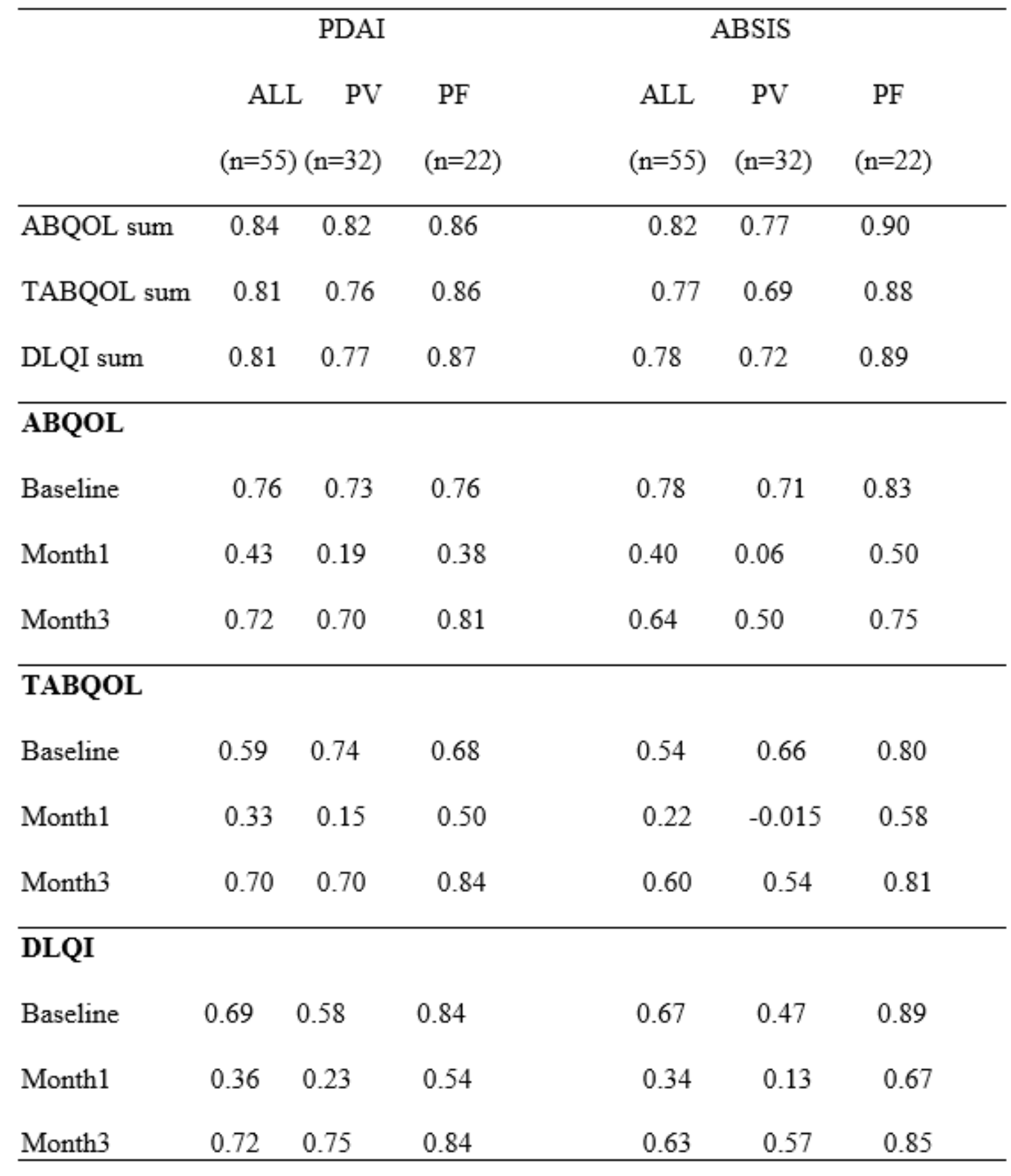

PNP $=1$, due to the small sample size. PNP data were not statistically significant.

\section{Figures}




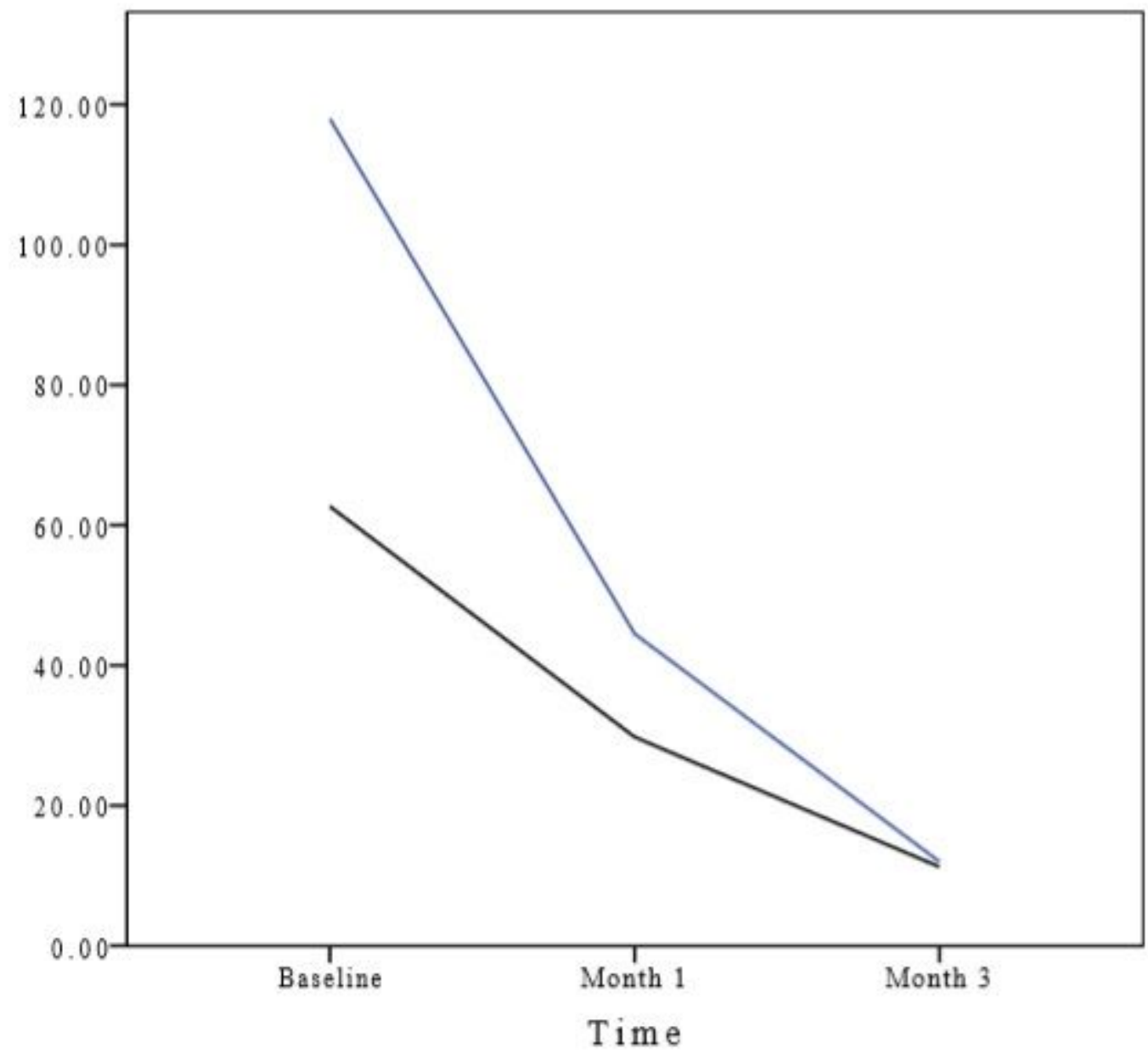

\section{Figure 1}

Mixed linear model for the changes of median QOL (ABQOL/TABQOL/DLQI) and disease severity (PDAI/BPDAI/ABSIS) in 85 Chinese patients with pemphigus at baseline, and 1 and 3 months after treatment. 


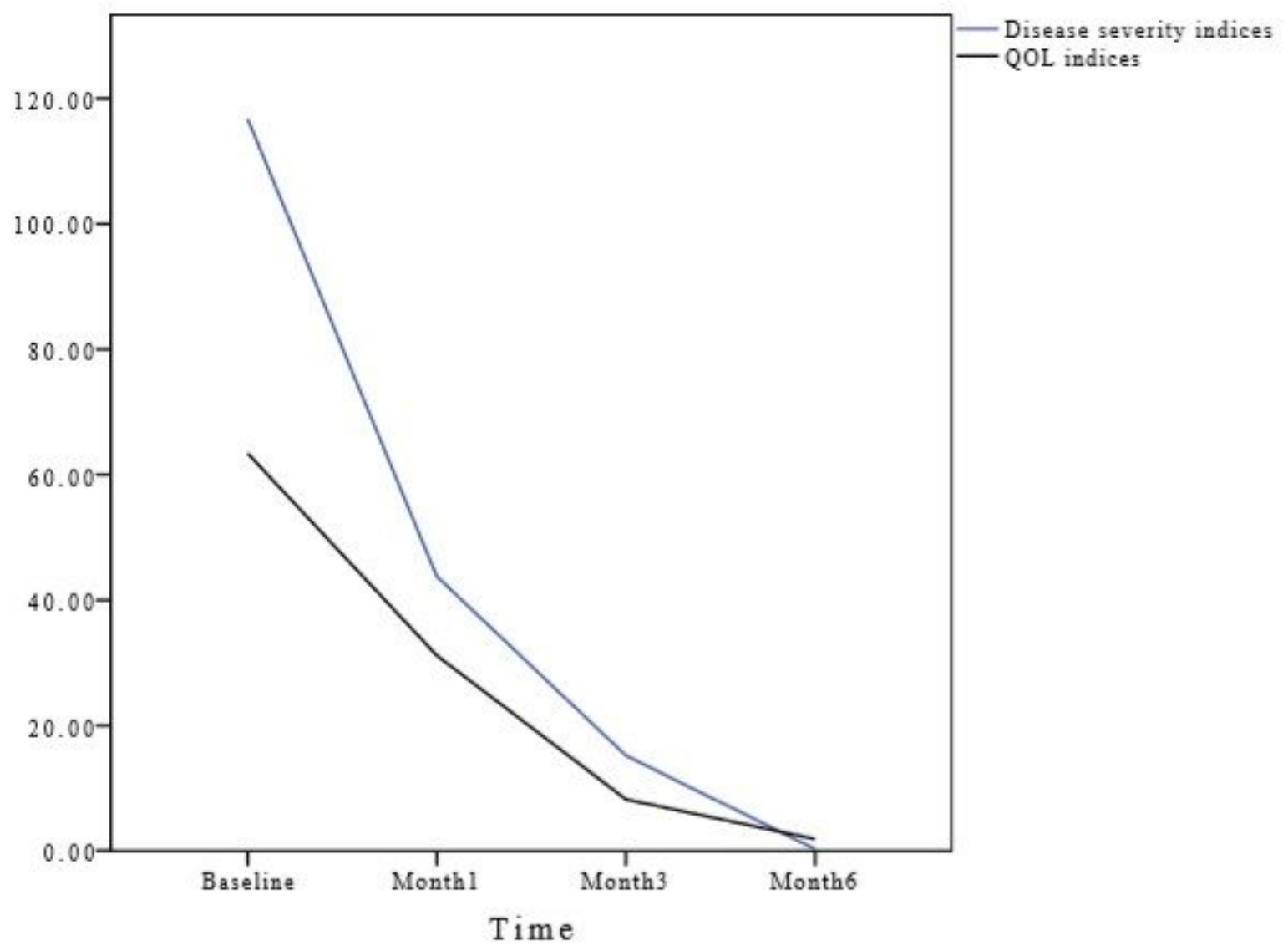

Figure 2

Mixed linear model for the changes of median QOL (ABQOL/TABQOL/DLQI) and disease severity (BPDAI/BPDAl, ABSIS) in 65 Chinese patients with BP at baseline, and 1, 3, and 6 months after treatment. 